山्山FFRANÇAISE

$\supset \mathrm{DE}$

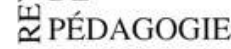

\section{Revue française de pédagogie}

Recherches en éducation

182 | 2013

Connaissances et politiques d'éducation : quelles interactions?

\title{
L'aide aux devoirs. Dispositif de lutte contre l'échec scolaire ou caisse de résonance des difficultés non résolues au sein de la classe?
}

Is homework help a programme to prevent school failure or does it bring attention to unsolved in-class difficulties?

Séverine Kakpo et Julien Netter

\section{(2) OpenEdition}

\section{Journals}

Édition électronique

URL : http://journals.openedition.org/rfp/4003

DOI : $10.4000 /$ rfp.4003

ISSN : 2105-2913

Éditeur

ENS Éditions

Édition imprimée

Date de publication : 28 août 2013

Pagination : 55-70

ISSN : 0556-7807

Référence électronique

Séverine Kakpo et Julien Netter, «L'aide aux devoirs. Dispositif de lutte contre l'échec scolaire ou caisse de résonance des difficultés non résolues au sein de la classe? », Revue française de pédagogie [En ligne], 182 | 2013, mis en ligne le 28 août 2016, consulté le 19 avril 2019. URL : http:// journals.openedition.org/rfp/4003; DOI : 10.4000/rfp.4003 


\title{
L'aide aux devoirs. Dispositif de lutte contre l'échec scolaire ou caisse de résonance des difficultés non résolves au sein de la classe?
}

\author{
Séverine Kakpo et Julien Netter
}

Cet article présente l'analyse d'un dispositif d'aide aux devoirs au travers des deux grands systèmes de contraintes auxquels il est soumis. L'étude du premier de ces systèmes nous permet de mettre en évidence différentes sources de tension venues de la classe (faible maîtrise des notions, opacité des consignes, etc.) qui se cumulent et dessinent une situation initiale très déterminée. Le second, propre au dispositif, découle des partis pris qui y sont mis en œuvre, en particulier de la forte individualisation du travail et du contexte de son organisation. Dans les quartiers populaires où nous avons enquêté, ces deux systèmes entrent en résonance et laissent peu de liberté aux intervenants, dès lors moins enclins à centrer leur action sur les apprentissages qu'à développer des stratégies de survie dont le bénéfice pour les élèves n'est pas toujours évident.

Mots-clés (TESE) : devoirs, école primaire, éducation extra-scolaire, niveau local, difficulté d'apprentissage, inégalité sociale.

Les politiques de lutte contre les inégalités sociales de réussite scolaire ont évolué au cours de ces dernières décennies vers ce que J.-Y. Rochex (2010) nomme "le troisième âge des politiques d'éducation prioritaire ", qui « voit s'affirmer non plus les problématiques de la compensation et de la lutte contre l'exclusion mais celle de la maximisation des chances de réussite de chaque individu ou catégories d'individus ". Le paysage contemporain des dispositifs de lutte contre l'échec scolaire reflète autant ce nouvel âge que les changements d'orientation qui y ont mené. En effet, selon une « logique d'empilement parfois vertigineuse » (Félix, Saujat \& Combes, 2012), les nouveaux dispositifs et les conceptions qui les sous-tendent se sont davantage agrégés à ceux déjà existants qu'ils ne les ont systématiquement remplacés. Au sein de cet ensemble composite, le champ de " l'accompagne- ment scolaire » (Glasman, 2001), qui s'est massivement développé depuis les années 1990, est resté principalement ancré dans une logique typique du " premier âge des politiques d'éducation prioritaire ", qui vise à " compenser les déficits ou carences dont souffriraient les enfants des catégories populaires " (Rochex, 2010) et qui tend - comme le souligne Bernstein (1975) - à détourner l'attention " de l'organisation interne et du contexte éducatif de l'école ", en la dirigeant "sur les familles et sur les enfants ", plutôt que de " mettre en question ou d'expliciter les présupposés sociaux impliqués dans la définition sociale du savoir légitime ou dans la mise en œuvre légitime de ce savoir ".

Les résultats des travaux qui se sont proposés, au moyen d'approches quantitatives, de mesurer l'effet des dispositifs d'accompagnement en matière de 
réduction des inégalités sociales de réussite scolaire (Glasman, 2001 ; Glasman \& Besson, 2004 ; Piquée, 2001, 2003 ; Suchaut, 2005) rejoignent en grande partie les bilans souvent décevants des politiques de lutte contre l'échec scolaire (Moisan \& Simon, 1997 ; Rochex, 1997 ; Chauveau, 1999 ; Armand \& Gille, 2006). Dans l'ensemble, les élèves pris en charge ne réalisent pas des progressions sensiblement différentes de celles des autres élèves (Piquée, 2001). Si les résultats de ces travaux ne permettent pas de conclure de manière définitive et générale à l'inefficacité de ces dispositifs, ils incitent fortement à se déprendre de l'évidence qui consiste à les croire nécessairement bénéfiques pour les élèves. Dans le cadre d'une recherche conduite au sein de l'équipe ESCOL ${ }^{1}$, nous nous sommes proposés d'étudier les logiques pédagogiques à l'œuvre au sein des dispositifs d'aide aux devoirs, qui sont généralement pensés comme un équivalent de ce que pourrait être l'encadrement du travail hors la classe dans les familles les plus favorisées. Sans viser nullement à produire des résultats en termes de causalité mécanique, nous avons cherché à éclairer les raisons pour lesquelles les élèves qui fréquentent ce type de dispositif ne réalisent pas toujours les progressions escomptées. Partant du principe que les bénéfices cognitifs qu'ils peuvent en retirer dépendent intrinsèquement de la nature de ce qui circule entre la classe et les dispositifs hors la classe, nous avons pris le parti d'articuler le plus étroitement possible l'analyse de l'activité des élèves et celle de leurs encadrants à l'analyse de ce qui est au cœur du fonctionnement pédagogique de ces dispositifs : les devoirs. Nous nous démarquons ainsi d'une conception "externaliste " qui chercherait à situer les raisons d'une faible efficacité de ces dispositifs du côté des caractéristiques individuelles des élèves, mais également d'une logique " internaliste » qui focaliserait exclusivement l'attention sur les caractéristiques de fonctionnement de ces dispositifs (type d'encadrants, taux d'encadrement, organisation structurelle, etc.). Nous concevons ce qui se joue au sein des aides aux devoirs comme fondamentalement inséré dans le cadre de la "boucle " pédagogique du travail des élèves (Rayou, 2009), constituée de la séance qui a eu lieu en classe sur le savoir, de l'activité dévolue à l'espace-temps des devoirs, de ce qui se déroule dans le temps de l'étude², du retour en classe et du traitement qui est fait alors du travail réalisé dans la boucle. Dans l'idéal, cette boucle permet la bonification des apprentissages à travers l'exportation de tâches en lien avec les contenus notionnels étudiés en classe.
Notre démarche s'inscrit, dans le prolongement des travaux conduits par P. Rayou (2009), au croisement d'une sociologie des "marges " de l'école qui a peu cherché à analyser de manière fine les processus cognitifs à l'œuvre au sein des dispositifs d'accompagnement (Glasman, 2001 ; Piquée, 2001, 2003) et d'une sociologie des inégalités d'apprentissage qui s'est principalement centrée jusqu'ici sur ce qui se joue au sein des classes (Lahire, 1993 ; Bautier \& Rochex, 2007 ; Bautier \& Goigoux, 2004 ; Bonnéry, 2007, 2009 ; Bautier \& Rayou, 2009 ; Rochex \& Crinon, 2011). Elle emprunte également des éléments à l'ethnographie interactionniste (Woods, 1997) ainsi qu'à une clinique de l'activité soucieuse de comprendre les dimensions potentiellement antagonistes de l'activité des acteurs (Clot \& Faïta, 2000 ; Goigoux, 2007).

Pour conduire cette recherche, nous avons enquêté sur un dispositif municipal d'aide aux devoirs ${ }^{3}$ implanté dans les divers quartiers d'une commune populaire de la périphérie parisienne (Granville ici). S'il présente des caractéristiques similaires à l'ensemble des structures de ce genre (Glasman, 2001), le dispositif granvillois présente quelques spécificités notables. II est exclusivement encadré par des enseignants, incités par une rémunération attrayante à s'y engager. Les élèves de primaire qui y sont accueillis sont donc systématiquement encadrés par des enseignants de leur école, parfois même par leur propre enseignant (le taux d'encadrement est de 1 enseignant pour 13 élèves). Le dispositif a par ailleurs été théoriquement conçu pour s'adapter à la " diversité des besoins " des enfants 4 . II prévoit en effet que les élèves « autonomes » ou qui ont besoin d'un "léger encadrement pour la réalisation de leurs devoirs " travaillent au sein de " groupes d'étude " d'une quinzaine d'élèves et que ceux qui rencontrent des " difficultés » travaillent au sein de " groupes restreints " constitués de huit élèves au maximum. Ces spécificités témoignent du fort volontarisme politique qui anime la municipalité en matière de lutte contre l'échec, mais aussi de l'importance des moyens financiers investis. Elles font du dispositif granvillois un terrain particulièrement intéressant dans la mesure où elles incitent à ne pas s'attarder sur la question des moyens et du mode d'organisation du dispositif (ce que la ville a fait jusqu'ici pour tenter de l'améliorer, ses résultats étant jugés globalement décevants) et à porter plutôt le regard vers les inter-relations entre les différents espaces-temps de la «boucle » du travail personnel des élèves.

Cette recherche nous a permis de mettre au jour l'existence de dysfonctionnements récurrents du système des devoirs, qui grèvent le fonctionnement du dispositif, lequel permet dès lors difficilement aux 


\section{Méthodologie}

Cherchant à étudier de manière fine les pratiques et les logiques d'action mises en œuvre à l'aide aux devoirs tout en ayant une vision d'ensemble du dispositif, nous avons fait le choix de procéder à une observation dynamique de l'activité des élèves et des enseignants, en circulant dans les salles auprès des différents acteurs en présence. Nous avons enregistré les échanges observés entre élèves et enseignants ainsi que les discussions informelles que nous avons nous-mêmes sollicitées dans le but de recueillir et d'analyser leurs représentations et raisons d'agir. Des photographies des cahiers de textes, cahiers, brouillons, fichiers, manuels, classeurs d'élèves ont également été réalisées de manière à appréhender finement les contenus d'apprentissage en jeu dans les situations observées. Nous avons enfin filmé les séances en fond de classe de façon à pouvoir bénéficier d'un regard périphérique fixe donnant à voir l'organisation générale de l'activité. Si le dispositif méthodologique mis en œuvre nous a permis d'explorer l'étude, il présente une limite importante : celle de ne pas nous avoir permis d'investir l'espace de la classe ni d'observer la circulation effective du travail entre classe et étude. Craignant que notre présence ne soit perçue par les enseignants comme une atteinte à leur liberté pédagogique, la municipalité n'a pas souhaité que nous nous aventurions sur ce terrain ${ }^{5}$. Pour chaque séance observée, nous avons donc cherché à reconstruire, depuis l'étude, la circulation des tâches entre classe et hors classe, en interrogeant sur le vif les élèves observés, mais aussi en questionnant les prescripteurs dès que l'occasion nous en était donnée. De ce point de vue, l'inscription durable de notre équipe dans le paysage des écoles de l'enquête ${ }^{6}$ a constitué un atout. Au final, l'enquête de terrain - qui s'est déroulée sur six mois consécutifs - nous a permis de recueillir un matériau conséquent : soixante-seize séances d'aide aux devoirs ont été observées (dont dix en " groupes d'étude restreints "). Plusieurs centaines de clichés de supports pédagogiques ont par ailleurs été réalisées.

encadrants d'aider les élèves à tirer un réel bénéfice cognitif de leur travail, quand il n'incite pas franchement les adultes à mettre en œuvre des « stratégies de survie » peu favorables aux apprentissages.

\section{DES DYSFONCTIONNEMENTS RÉCURRENTS DE LA « BOUCLE » QUI PÈSENT SUR L'ÉTUDE}

\section{L'irrégularité du volume des devoirs}

Le premier type de dysfonctionnement que I'on observe à l'étude est lié à la quantité de travail prescrit. Cette valeur est éminemment relative puisqu'elle dépend intrinsèquement du degré de maîtrise que les élèves ont des notions en jeu dans leurs devoirs. Nous avons cependant cherché à en donner une mesure objective car le débit de la boucle et ses variations déterminent en grande partie les capacités des enfants à répondre aux attentes de l'institution et celles du dispositif à atteindre son objectif : renvoyer les élèves chez eux avec des devoirs « faits et corrigés »?

Les enseignants prescrivent régulièrement des devoirs mais ils le font dans des proportions qui varient significativement d'un jour à l'autre et qui, rapportées à un temps d'étude qui lui ne varie pas, peuvent parfois sembler relativement inadaptées. Nous avons relevé un échantillon de cahiers de textes d'élèves de CE1 qui ne peut prétendre être représentatif mais fournit quelques indicateurs significatifs. Sur un total de cent quatrevingt-dix-neuf jours de prescription étudiés (hors veilles de vacances), nous avons mesuré la quantité de tâches à réaliser chaque jour (une tâche peut être un exer- cice, une leçon, une lecture, etc. $\left.{ }^{8}\right)$. Si, dans $15 \%$ des cas, les élèves ont une seule tâche à effectuer, dans près de $40 \%$ des cas, ils en ont trois ou plus. Ainsi peut-il leur être demandé un soir pour le lendemain d'effectuer une lecture découverte d'un texte, de traiter par écrit six questions de compréhension et de faire encore deux exercices de mathématiques ${ }^{9}$. II s'agit pour des élèves de CE1 de prescriptions conséquentes, qui augmentent significativement leur journée de travail. Par ailleurs, contrairement à ce que pourrait laisser penser la volonté parfois affichée par les enseignants de ne pas surcharger les élèves de travail du jour pour le lendemain, nos observations montrent qu'il n'existe généralement pas de lien significatif entre le jour de la prescription et la quantité de tâches données.

Les intervenants se plaignent donc régulièrement de la quantité de travail donné par certains de leurs collègues. L'un d'eux nous confie même qu'il a pour habitude de consulter l'une de ses collègues avant l'étude afin que celle-ci lui indique les tâches à traiter en priorité. Ces prescriptions parfois importantes s'imposent à tous les élèves quelles que soient les difficultés qu'elles représentent pour eux. Mais il existe même parfois un " effet de traîne " qui augmente de manière significative la quantité de tâches auxquelles sont confrontés les élèves les plus fragiles scolairement. Estimant que ceux-ci n'ont pas suffisamment appris leur leçon, certains enseignants leur redonnent en effet le travail à faire pour le lendemain, en plus des devoirs du jour donnés à l'ensemble de la classe. Le nombre de tâches peut par ailleurs augmenter nettement dans le cas de révisions avant évaluation, voire atteindre des proportions qui explosent littéralement le cadre temporel 
de l'étude. Un mardi soir, par exemple, les CE1 ont huit leçons de grammaire à "réviser " et huit mots à " apprendre » en vue d'une dictée. À l'opposé de ces sommets, il arrive, très rarement mais cela arrive néanmoins, que les enfants n'aient pas de devoirs ou plus fréquemment qu'ils n'aient qu'une tâche à réaliser. Les études dans lesquelles ils se trouvent paraissent alors bien peu adaptées au désœuvrement qui les gagne rapidement et l'ambiance de travail, quand le cas se présente, pâtit de cette situation dans la seconde moitié de l'étude. Le trop-plein et le trop-peu de tâches constituent donc autant de variations qui contribuent à peser sur le fonctionnement de l'étude.

\section{Des prescriptions qui renvoient à des notions insuffisamment maîtrisées}

Le second dysfonctionnement repéré tient au fait que les devoirs renvoient souvent à des notions n'ayant pas été suffisamment acquises en amont par les élèves. Travailler dans une réelle autonomie intellectuelle (Lahire, 2001) est alors inaccessible pour les élèves, généralement contraints de solliciter auprès des intervenants des restaurations conceptuelles de fond (soit en début d'étude soit au moment de la vérification du travail effectué). Ces prescriptions contribuent inévitablement à rendre le dispositif moins efficace qu'il ne pourrait l'être, voire à le détourner de sa fonction, en le transformant en " annexe » de la phase d'enseignement au sens strict.

En confrontant les données que nous avons pu recueillir auprès des prescripteurs avec celles tirées de nos observations, nous avons pu identifier différents types de dévolution à l'étude du travail qui devrait normalement relever de la classe. La première consiste à exporter vers l'étude des devoirs dont les enseignants jugent sincèrement mais à tort qu'ils renvoient à des notions acquises. Cette dévolution involontaire semble être souvent liée au fait que les enseignants font de la classe un espace qui prend en charge le seul objectif d'identification des savoirs mais qui ne permet pas aux élèves de s'en saisir et de réinvestir les notions découvertes (Bonnéry, 2007 ; Rochex \& Crinon, 2011 ; Zakaria, 2012). Ces pratiques font donc de l'étude un espace de première mise en application et vérification de la bonne appropriation de ces savoirs. Or, celle-ci révèle souvent que les élèves ne se sont pas appropriés les notions en jeu. C'est ce que remarque par exemple un enseignant ayant encadré ses propres élèves à l'étude et s'étant rendu compte qu'aucun n'avait compris la leçon vue quelques heures plus tôt : "en classe, ils ont l'air de comprendre mais en fait ils n'impriment pas ».
Une seconde forme consiste pour les enseignants à expédier vers l'étude des tâches dont ils savent pertinemment qu'elles vont mettre en difficulté leurs élèves et les intervenants par la même occasion. "Tu vas voir tu vas t'amuser parce qu'il y a la moitié qui n'a strictement rien compris! ", déclare par exemple une enseignante à l'un de ses collègues. Si ces prescripteurs sont capables d'anticiper les difficultés de leurs élèves, c'est parce qu'ils se sont parfois efforcés de vérifier en classe l'acquisition des notions. Mais contrairement à ce que préconiserait le fonctionnement idéal de la boucle, la mise au jour du « raté » d'enseignementapprentissage n'a pas entraîné la suspension de la circulation : des devoirs sont quand même prescrits. Dans d'autres cas, c'est un simple manque de temps qui est à l'origine de ces prescriptions prématurées. On peut faire l'hypothèse que la prescription de ce genre de tâches sert à externaliser une partie des tensions et des contradictions qui n'ont pas pu trouver leur issue en classe et à normaliser ce qui s'y produit. Donner des devoirs, n'est-ce pas une manière de signifier aux yeux de tous que l'institution a fait sa part de travail et qu'il revient aux élèves d'assumer la leur?

Une troisième forme de dévolution consiste pour l'enseignant à externaliser une tâche dont il sait qu'elle va mettre en difficulté ses élèves - puisque l'externalisation intervient alors que la notion vient à peine d'être abordée - mais à le faire volontairement, en misant sur les effets bénéfiques de cette confrontation précoce. Le bain cognitif qu'induit la prescription de ce type de devoirs est censé rendre plus efficace la phase d'appropriation qui aura lieu le lendemain en classe et sera ensuite suivie d'une phase d'externalisation plus conventionnelle. L'externalisation de ces tâches prématurées s'assume donc comme démarche pédagogique comme en témoignent les propos de ce prescripteur : "Qu'il sache quand même de quoi on parle, je pense que ce sera plus efficace quand on va travailler plus précisément. Le fait de revoir la leçon même s'il comprend pas tout, qu'il se souvient pas forcément, ça aura été dans un petit coin de sa tête... Je suis pas très ambitieux au début, je me dis que c'est toujours ça de pris pour demain. »

\section{Opacité des prescriptions : consignes et supports pédagogiques}

La relative opacité des consignes et des supports pédagogiques auxquels sont confrontés les élèves, tout particulièrement lorsqu'elle se conjugue au fait que les devoirs renvoient à des notions non acquises, contribue à saper la circulation du travail dans la boucle. Cette 
opacité met en difficulté les élèves, y compris ceux dont la maîtrise des notions est peu ou non problématique, et constitue un problème majeur pour l'étude, parce qu'elle contraint les intervenants à se lancer dans un travail d'explication parfois très chronophage.

Les recherches consacrées aux consignes insistent sur le décalage qui existe entre la compréhension littérale des consignes et la compréhension des enjeux qui leur sont sous-jacents. Cette seconde opération est délicate car les consignes se caractérisent fréquemment par un fort degré d'implicite, qui se prête à bien des malentendus socio-cognitifs (Bautier \& Rochex, 2007 ; Bonnéry, 2007 ; Bautier \& Rayou, 2009). En classe, le face à face, souvent solitaire, entre l'apprenant et la consigne de travail (Zakhartchouk, 2000) est généralement suivi d'un travail décisif de reformulation et de contextualisation par les enseignants (Zerbato-Poudou, 2001). Dans le cas du travail hors la classe, l'absence de l'enseignant et donc de guidage face à la consigne accroît la difficulté pour les élèves à s'en saisir dans le sens attendu par les prescripteurs (Félix, 2002 ; Barrère, 2003 ; Glasman \& Besson, 2004 ; Kakpo \& Rayou, 2010).

Notre enquête confirme qu'une grande ambiguïté affecte les termes génériques les plus couramment employés pour prescrire des devoirs. C'est par exemple le cas du verbe "apprendre » qui se retrouve un jour sur deux en moyenne dans les cahiers de textes étudiés. Les enseignants prescripteurs peuvent en attendre des activités cognitives bien différentes. II peut en effet s'agir d'" apprendre par cœur ", de "fixer ", d'« être capable d'appliquer ", d'" être capable de redire avec ses propres mots ", de "savoir réécrire sans modèle " ou d'un mélange de plusieurs de ces acceptions. Sous son apparente évidence, le terme relève donc d'un " cadrage faible de l'activité " (Bernstein, 2007) qui risque fort de conduire les élèves à ne pas sélectionner le sens spécifique adéquat du terme générique. Les adultes de l'étude sont eux-mêmes confrontés à cette opacité. Alors que nous observons des élèves tentant de décrypter une consigne particulièrement ambiguë (« apprendre les mots »), nous constatons par exemple que l'intervenant, perplexe, en confirme les interprétations contradictoires des uns et des autres (« mémoriser la définition », " savoir reformuler ", " mémoriser l'orthographe »).

La confiance que les enfants portent à leurs propres capacités joue un rôle déterminant dans l'autorisation qu'ils s'octroient ou non d'interpréter la prescription, et par là même de prendre du recul par rapport à la commande institutionnelle. On pense ici aux analyses de C. Félix (2002) qui montrent que les élèves qu'elle appelle "faibles » peuvent se retrouver piégés par leur lecture littérale des prescriptions, parfois peu appropriée, quand
Figure 1. Un support opaque

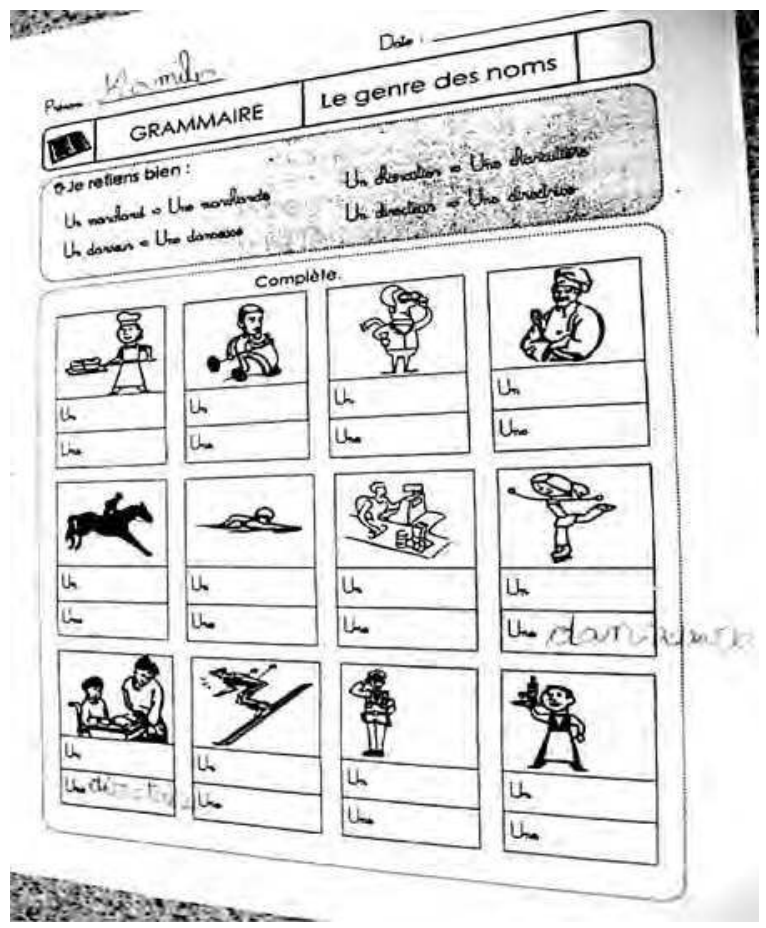

Note : dans l'encadré « Je retiens bien », lire la liste d'exemples suivante: Un marchand > Une marchande; Un danseur > Une danseuse; Un charcutier > Une charcutière ; Un directeur > Une directrice.

les élèves « forts » savent prendre des libertés avec elle. La prescription agit donc comme un élément de renforcement des inégalités et nous semble relever de pratiques de " sous-ajustement ", les élèves étant " confrontés à des tâches et des situations trop ouvertes, à des contrats et des milieux didactiques trop flous et trop larges, pour le traitement desquels les plus démunis d'entre eux ne peuvent mobiliser que leurs expériences premières du monde, sans pouvoir disposer ou faire usage d'aides ou de critères leur permettant de redéfinir les tâches de manière pertinente " (Bautier \& Goigoux, 2004).

En l'absence d'une consigne claire, le support de travail des élèves, qu'il s'agisse d'une leçon ou d'un exercice, pourrait leur permettre d'inférer l'activité attendue. Pourtant, l'abord des supports que nous avons rencontrés se révèle extrêmement délicat pour une grande partie des élèves. Ils éludent en effet le plus souvent les informations qui permettraient de se diriger dans la structure de la leçon ou d'identifier l'enjeu d'apprentissage que recèle l'exercice. Ainsi, dans une de nos observations, Kamila (CE1) se révèle incapable d'interpréter la consigne " complète " qui figure sur sa fiche (voir figure 1). 
Celle-ci ne prend véritablement sens qu'à condition d'être mise en relation avec deux autres éléments : le titre de la leçon et les quatre exemples donnés à observer sans autre forme de commentaire que « Je retiens bien ». L'opacité est frappante : on ne trouve aucune mention des termes « masculin » et «féminin ", qui sont pourtant au cœur des mobilisations attendues ici. Après avoir tenté de relier par des flèches les exemples et les illustrations (l'ambivalence des dessins rendant possible cette interprétation), Kamila s'engage finalement dans un jeu de devinettes sur les métiers, privilégiant - comme l'y incitent les caractéristiques du support - la mobilisation de ses connaissances pragmatiques sur le monde (Lahire, 1993). Dans la foulée, elle évoque d'ailleurs avec son camarade de table son dernier passage chez le boulanger puis le souhait de sa sœur de devenir chanteuse.

L'explicitation de ces " cadrages » flous n'est manifestement pas suffisamment prise en charge au sein de la classe, car les élèves qui ne partagent pas l'évidence scolaire de la nécessité d'un décryptage peinent à reconstruire la logique d'ensemble de ces architectures complexes et tendent à mobiliser les raisonnements et savoirs qui leur semblent les plus évidents, souvent loin des activités attendues. Nos résultats convergent en ce sens largement avec ceux de plusieurs recherches (Vigner, 1997 ; Bautier, 2010 ; Bautier, Crinon, Delarue-Breton et al., 2012 ; Bautier, Bonnéry \& Kakpo, 2013) qui montrent comment les supports se sont historiquement transformés depuis les années 1950, passant de textes essentiellement linéaires à des supports " composites ", où les informations sont délivrées de manière multiple et discontinue, et qui contraignent les élèves à réaliser un travail très délicat de mise en relation d'éléments sémiotiques et langagiers variés puis à la reconstruction d'un discours linéaire qui les synthétise. La nécessité récurrente d'une interprétation de la prescription pèse sur les premiers instants de l'étude, moment où les intervenants doivent s'assurer que tous les élèves savent ce qu'ils ont à faire, mais également sur l'ensemble de son déroulement, les interprétations des élèves se révélant souvent erronées.

\section{La fragmentation des savoirs et des supports de travail}

Au-delà de la consigne, l'identification de l'activité attendue passe par son rattachement à "I'univers social des tâches scolaires " (Brossard, 1993). II nous semble que la capacité à opérer ce rattachement dépend en partie du type de situations rencontrées.
Ainsi, lorsque des élèves de CM1 doivent apprendre un poème, ils n'ont aucun mal à interpréter la consigne parce qu'ils ont déjà appris des poèmes et identifient la prescription comme une demande de mémorisation. Au-delà de la simple mise en lien terme à terme de deux situations, la " conscience disciplinaire " (Reuter, 2007) que les élèves ont forgée par leur expérience scolaire nous paraît ici fondamentale parce qu'elle leur permet de structurer ces associations. C'est alors le lien entre la tâche prescrite et l'ensemble des activités menées en classe auxquelles elle est censée référer qui est en jeu. Nous allons montrer qu'une fragmentation exacerbée caractérise les devoirs - bien au-delà de la « fragmentation » des tâches traditionnellement opérée par l'école (Perrenoud, 1995) -, qui tend à distendre ce lien en dissociant notamment les notions de leurs contextes d'utilisation, d'un point de vue aussi bien cognitif que matériel.

Les cent quatre-vingt-dix-neuf jours de prescription que nous avons étudiés dans les cahiers de textes de CE110 témoignent de la fragmentation cognitive que l'on y trouve. En moyenne, les élèves sont confrontés à plus de deux tâches par jour, relevant de deux disciplines distinctes, et comprenant un exercice et une leçon. Le quart seulement des exercices est prescrit le même jour que l'apprentissage des leçons ou que les lectures nécessaires à leur réalisation. Les élèves ont donc généralement une leçon à apprendre et un exercice à réaliser qui n'ont rien à voir l'un avec l'autre. Lorsqu'un lien est avéré, il n'est explicité que pour $7 \%$ seulement du total des exercices prescrits, avec une consigne du type "en t'aidant de la leçon... ». Ce peu d'insistance pose problème, car nos observations montrent que lorsque le lien n'est pas explicite les enfants ont tendance à faire d'abord leurs exercices et à s'occuper des leçons ensuite, ces dernières étant peut-être jugées plus compliquées et leur intérêt moins évident (Glasman \& Besson, 2004). Bien que l'enseignant pense avoir prescrit une leçon et l'exercice d'application qui lui correspond, ses élèves réalisent l'exercice sans connaître leur leçon ni penser à s'y référer, puis apprennent péniblement leur leçon sans vraiment savoir à quoi elle peut leur servir.

À cette fragmentation cognitive correspond un éclatement matériel des supports de travail qui la renforce. Le modèle d'organisation le plus couramment observé au sein des études articule outils traditionnels non disciplinaires (cahier du jour, cahier de devoirs où les élèves écrivent et s'exercent) avec des supports régis par les grandes catégories disciplinaires matérialisées par le " code couleur » des protège-cahiers, en particulier pour le stockage des leçons. La profusion 
des supports, la désolidarisation des lieux servant à s'exercer et des lieux servant à conserver les leçons, la complexité des modes de classification disciplinaire adoptés et enfin le caractère peu évident de la codification utilisée sont autant d'éléments qui concourent à la fragmentation cognitive, d'autant plus qu'ils entraînent chez de nombreux élèves des difficultés pour se munir des bons supports de travail. C'est le cas de cette élève de CE2 que l'intervenant de l'étude décrit pourtant comme une «bonne élève ". Elle constate qu'il lui manque son " cahier bleu » (de mathématiques) nécessaire à l'apprentissage de sa leçon « calcul/Cal4 ». Or, dit-elle, c'est dans ce cahier que se trouve Cal4. En effet : "C'était sur la numération ça c'est sûr ! [...] parce que tout ce qui est dans le cahier bleu foncé, eh ben, c'est géométrie, numération, grammaire... ". Les résultats de nos observations convergent ainsi largement avec ceux des travaux d'A.-M. Chartier et de P. Renard (2000), qui mettent en évidence l'effacement progressif du « modèle primaire ancien ", construit autour d'un cahier unique organisé par la chronologie des exercices scolaires au profit d'un «modèle secondaire ». Mais la " classification" à laquelle ce nouveau modèle introduit par la pratique (Chartier, 2003) apparaît moins maîtrisée par les élèves que supposée acquise par les enseignants.

Ce morcellement cognitif et matériel présente une difficulté. II rend l'activité prescrite peu lisible et transfère largement aux élèves la responsabilité de tisser le lien entre la prescription et la classe. II leur impose en outre l'anticipation de ce tissage pour constituer en classe le cartable qui contient tous les supports dont ils auront besoin à l'étude pour les différentes tâches qui leur sont prescrites. Les élèves que nous avons observés s'orientent finalement peu dans cet univers scolaire fragmenté, la "classification " ne pouvant dès lors suppléer la faiblesse du " cadrage " des prescriptions pointée dans la sous-partie précédente (Bernstein, 2007). Et pour certains, qui peinent à construire une cohérence d'ensemble, la continuité des apprentissages n'existe tout simplement pas, la consistance du lien cognitif et matériel entre la classe et l'étude étant trop incertaine. Les situations ne s'agencent alors plus, elles se juxtaposent, et les élèves les subissent plus qu'ils ne s'en saisissent (Brossard, 1994 ; Bautier \& Rochex, 2007), se révélant peu à même de rattacher l'activité attendue à une activité connue. Nos observations confirment sur ce point l'analyse de C. Félix (2002), selon laquelle on touche ici à une distinction essentielle entre les élèves "forts " et «faibles ». La difficulté se transforme alors rapidement en une contrainte pour les intervenants. En début de séance, on observe ainsi presque toujours qu'au moins un élève n'a pas tous les supports pour faire ses devoirs, malgré les indications orales qui ont pu être fournies en classe. Cela retarde la mise au travail car les intervenants doivent prendre le temps d'organiser leur laborieuse récupération dans le cartable puis dans l'enceinte de l'école, et sont dès lors peu disponibles pour mobiliser les autres élèves. Ils sont également contraints de se lancer dans un travail de " couture cognitive " qui retisse leçons et exemples, exercices et rappels notionnels, et peut finir par les accaparer totalement, en particulier lorsque les notions sont peu maîtrisées.

\section{Quand la boucle « tourne à vide »}

Nous avons montré que la façon dont les devoirs sont prescrits ne favorise pas leur inscription dans une boucle bénéfique et pèse lourdement sur le fonctionnement de l'étude. II existe pourtant un type de prescriptions qui relève de pratiques de "sur-ajustement » (Bautier \& Goigoux, 2004) et, faisant l'économie des problèmes de circulation, simplifie la tenue de l'étude. Pour autant, il nous semble problématique parce qu'il se révèle " particulièrement propice aux effets de leurre chez les élèves faibles, et conduit à considérer que "tout va bien" dès lors que les élèves parviennent à réussir les tâches proposées " (Bautier \& Goigoux, 2004). En facilitant le déroulement de l'étude, ce type de prescriptions contribue donc paradoxalement à en compromettre l'efficacité à long terme. Nous en décrirons deux variantes selon qu'elles sont directement conçues sans réels enjeux d'apprentissage, ou qu'elles en sont vidées a posteriori.

Ces prescriptions mettent en jeu des tâches qui ne sont pas directement inscrites dans les réseaux de notions étudiés en classe et ne les enrichissent donc pas. Par exemple, un soir, des élèves de CE2 doivent "savoir " les définitions de onze mots disparates sans lien entre eux ni avec aucun contexte textuel (topinambour, satellite, gyrophare, pachyderme, etc.). Ces « mots du jour ", extraits d'un calendrier-jeu, sont dévoilés chaque matin par le maître de la classe lors d'une forme de rituel : les élèves doivent deviner leur sens, puis copier leur définition. Au bout d'une période donnée, ces mots constituent une liste que les élèves doivent " savoir ». À l'étude, ils s'accommodent de cette tâche : ils peuvent réaliser leurs devoirs "en autonomie " dès lors que la consigne est explicitée et se mettre ainsi en règle avec la demande institutionnelle. II n'y a pas de problème de continuité parce qu'il n'y a pas réellement de continuité, la prescription n'entrant pas en résonance avec les notions étudiées 


\section{Singulier $\rightarrow$ Pluriel}

\section{3}

\section{Un ou des devant un nom}

\section{le plus souvent $s$ au pluriel}

Un garçon écoute la radio. $\rightarrow$ Des garçons écoutent la radio.

le.

mon, ton, son

ce

Une fille écoute la radio.

la.

ma, to sa.

cette

\section{- quelquefois $x$}

$$
\begin{array}{ll}
\text { un château } & \rightarrow \text { des châteaux } \\
\text { un cheval } & \rightarrow \text { des chevaux } \\
\text { un travail } & \rightarrow \text { des travaux } \\
\text { un chou } & \rightarrow \text { des choux } \\
\text { un jeu } & \rightarrow \text { des jeux }
\end{array}
$$

\section{! une souris $\rightarrow$ des souris un prix $\rightarrow$ des prix un nez $\rightarrow$ des nez}

par ailleurs en classe. En évacuant la nécessité d'une circulation, une telle prescription facilite donc le fonctionnement de l'étude, mais semble assimilable à une tâche de « bas niveau cognitif » (Bautier \& Goigoux, 2004).

D'autres fois, c'est la façon dont une prescription est formulée qui transforme une tâche a priori susceptible d'être reliée à un contenu notionnel étudié en classe en une « tâche de bas niveau ». L'une de nos observations, qui met aux prises des élèves de CE1 avec une leçon sur le pluriel des noms, en fournit un bon exemple. Cette situation est a priori très différente de la précédente parce que le travail prescrit est fortement en lien avec des contenus étudiés en classe : les notions de pluriel et de nom. Mais la leçon, photocopiée à partir d'une fiche, est d'un abord très difficile parce qu'elle ne présente à aucun moment les notions sous une forme claire, que les règles d'accord sont présentées de manière allusive et que l'architecture d'ensemble de la leçon est complexe (voir figure 2).
L'opacité de ce support est conjuguée à une prescription très floue consignée sur le cahier de textes, « apprendre la leçon ». Aucune référence n'est faite par ailleurs à des exercices réalisés en classe sur le pluriel. Les élèves disposent donc de peu d'indications pour se saisir de l'activité attendue. L'enseignant de la classe nous apprend avant l'étude qu'il a introduit oralement une seconde consigne qui double la demande écrite d'“ apprentissage » et tombe à point nommé pour dégager les élèves de cette difficulté : il a attiré l'attention des élèves sur les mots consignés en fin de leçon et leur a demandé de s'entraîner à les écrire pour mémoriser leur orthographe. Ces mots donnent un aperçu (mais cela n'est indiqué nulle part explicitement) des grandes familles de mots qui ont un pluriel en -x, en exception à la règle générale du pluriel en -s. En leur proposant une tâche facile à réaliser et ne nécessitant en rien la connaissance ou la compréhension de la leçon, la consigne orale rend possible la réalisation des devoirs en supprimant la nécessité de construire une continuité. II s'agit en quelque sorte d'un « déplacement » de la tâche d'apprentissage qui 
la vide de ses enjeux. II apparaît que les six élèves de la classe présents à l'étude se lancent dans la copie ou la dictée des dix mots prenant un pluriel en -x. Aucun ne perçoit l'architecture de la leçon ni le statut de ce qu'il est en train d'essayer de mémoriser, aucun ne se penche sur la règle du cas général. Du reste, le lendemain, lorsque l'enseignant effectue une évaluation pour vérifier que les devoirs sont bien appris, il dicte une série de mots extraits de la leçon qui tous font leur pluriel en -x, ce qui est conforme à l'interprétation que les élèves ont faite de la tâche.

De telles prescriptions sont adaptées à l'exigence d'« autonomie " sur laquelle le système des devoirs est fondé et ne posent en apparence pas de problème à l'étude. Mais elles nous semblent présenter un double écueil : elles constituent à la fois un masque et un leurre dangereux. En effet, si les tâches n'ont pas de rapport avec des notions construites en classe, elles ne permettent pas aux élèves de comprendre qu'il leur faut chercher les enjeux cognitifs sous-jacents aux activités scolaires. Comment en effet pourraient-ils chercher des enjeux qui ne sont pas présents ? En ce sens, elles masquent ce que l'on pourrait souhaiter voir au contraire mis en lumière. De telles tâches légitiment en outre l'interprétation erronée que ces enfants peuvent se construire des activités, conçues comme une succession de tâches (Bautier \& Rochex, 2007). En facilitant le travail des élèves à court terme dans le cadre de l'étude, on en induit donc certains en erreur sur la véritable nature de l'activité scolaire. Émerge alors un curriculum parallèle de bas niveau, source à terme d'amères désillusions (Bonnéry, 2007 ; Rochex \& Crinon, 2011).

Notre approche du système des devoirs, qui tient compte de leur insertion dans une boucle, permet d'éclairer les problèmes qui se posent à l'étude. Les difficultés que nous avons identifiées ne sont pas omniprésentes, elles ne se posent pas de la même façon à tous les enfants, elles sont cependant largement majoritaires dans notre corpus. Ce sont l'accumulation de ces difficultés et leur agencement qui posent problème. Le pilotage du dispositif suppose que $80 \%$ des élèves de l'étude sont « autonomes " ou " n'ont besoin que d'un léger encadrement pour faire leurs devoirs » et que seuls $20 \%$ d'entre eux ont véritablement besoin d'être " aidés "11. Or nos observations montrent que, confrontés à leurs devoirs, de nombreux élèves - généralement désignés comme «peu autonomes »-se trouvent dans l'incapacité de démarrer seuls leur travail et sont rapidement conduits à solliciter l'aide d'un adulte tandis que d'autres, tout en présentant l'apparence d'élèves " autonomes ", s'engagent dans un travail qui n'est pas celui permettant de s'approprier les savoirs scolaires au regard des exigences implicites. Nos résultats mettent donc à mal le postulat d'« autonomie » de la majorité des élèves devant la prescription, sur lequel reposent le système des devoirs ainsi que le dispositif "étude ". Ils permettent de comprendre pourquoi les écoles de la commune n'adoptent pas l'organisation prévue par la municipalité.

Nos résultats soulignent également le risque d'une focalisation sur une autonomie de façade qui serait en quelque sorte " naturelle » au détriment d'une " autonomie intellectuelle " (Lahire, 2001) rendue très difficile en contexte par les conditions de la prescription. L'étude est par conséquent confrontée à une contrainte initiale très forte qui, conjuguée aux contraintes propres du dispositif, peut pousser les intervenants à privilégier l'autonomie de façade sur l'autonomie intellectuelle. Nous allons à présent tenter de cerner la réalité de ce risque.

\section{ENTRE LES DYSFONCTIONNEMENTS DE LA BOUCLE ET LES CONTRAINTES DU DISPOSITIF}

\section{Une logique d'intervention centrée sur les enjeux d'apprentissage}

Le fonctionnement incertain de la boucle des devoirs constitue une trame qui conditionne ce que les intervenants peuvent faire dans le dispositif, mais ne le détermine pas mécaniquement. Parmi les différentes réponses que les intervenants mettent en oeuvre, nous avons constaté que des logiques d'intervention centrées sur l'activité permettent le rétablissement d'une certaine fluidité dans la boucle. Elles consistent essentiellement à faire percevoir aux élèves les enjeux d'apprentissage sous-jacents à la situation à laquelle ils sont confrontés, en la " décontextualisant " et "recontextualisant " (Vygotsky, [1934] 1985). Une telle démarche offre l'avantage d'intégrer la classe et le hors classe, permettant la circulation des contenus d'apprentissage. Elle contraint cependant les intervenants à s'inscrire dans une action sur le temps long, qui entre parfois en contradiction flagrante avec l'objectif institutionnel à court terme d'achèvement des devoirs, et avec la demande tant des prescripteurs que des parents d'élèves (Thin, 1998 ; Kakpo, 2012). Elle témoigne donc en outre d'un glissement de la situation d'aide aux devoirs vers une situation d'apprentissage. Elle suppose que les intervenants adhèrent au postulat d'éducabilité des élèves, à l'opposé des 
tentations récurrentes de naturalisation des difficultés, et qu'ils développent des "gestes professionnels " complexes (Clot \& Faïta, 2000 ; Goigoux, 2007). Ils doivent ainsi identifier les notions en jeu, les manipuler (c'est-à-dire mettre en œuvre le processus de décontextualisation et recontextualisation), déterminer une façon de les enseigner, analyser les erreurs des élèves pour identifier la source de leurs difficultés. On le voit, adopter une logique d'intervention centrée sur l'activité implique pour les intervenants à la fois une grande finesse d'exécution et un positionnement exigeant, pour le maintien duquel ils peuvent être contraints à une grande dépense d'énergie. Mais on ne peut parler de l'action des intervenants sans évoquer les contraintes issues du dispositif des études lui-même. Nous verrons alors que la mise en place de " situations d'enseignement " est largement entravée par des contraintes propres au dispositif qui mettent en jeu la fatigue et l'engagement des élèves, ou l'organisation fortement individualisée du travail.

\section{Des contraintes propres au dispositif}

S'inscrire dans une logique d'enseignement n'est pas neutre pour les intervenants. Cela implique de façon symétrique pour les élèves un effort qui est loin d'être négligeable. En effet, accepter de se confronter à ses difficultés d'apprentissage implique d'envisager une forme de transformation de soi et constitue donc une prise de risque individuelle (Vygotsky, [1934] 1985 ; Bautier \& Rochex, 2007). En outre, la fatigue des élèves est tangible après une pleine journée de classe, si bien que tout engagement supplémentaire dans une activité scolaire est avant tout perçu sous son aspect chronophage et génère rapidement des tensions. Les élèves préfèrent donc presque toujours la mise en conformité rapide avec la demande institutionnelle et inclinent les intervenants à se concentrer sur la réalisation rapide de la tâche.

On pourrait qualifier cette tendance de " courant contraire " parce qu'elle s'oppose à la circulation du travail dans la boucle. Elle est renforcée en ce sens par le mode d'organisation collectif de l'étude. La contrainte majeure qui en émane, perçue avec acuité par les intervenants, réside dans le manque de temps disponible pour mettre en place une logique d'action centrée sur l'activité :

Intervenant : «Par exemple si je prends le cas de Jordan... II avait strictement rien compris à ce qu'il fallait faire! II avait rien compris même à la notion de multiple ! Donc là, ben il faut prendre dix minutes, cinq minutes, et puis expliquer ce que c'est parce que... Sinon, on fait l'exercice à sa place en fait... Même si on l'aide ponctuellement sur l'exercice y va rien comprendre... Faut tout reprendre avec lui, le problème c'est qu'on peut pas tout reprendre avec les quinze enfants parce que... C'est pour ça que c'est assez complexe... [...] Par exemple, des fois, le mardi, on est... c'était la semaine dernière, on avait dix-huit gamins dans chaque étude. C'est infernal à gérer! »

II est facile de mesurer un "temps de l'étude " objectif et unique, a priori égal à une heure, qui court depuis l'arrivée des élèves jusqu'à leur départ de la salle d'étude. Dans les faits, on doit en déduire une série de petits moments liés à la mise en rang, à l'appel, aux circulations dans les escaliers, à la répartition et à l'installation des élèves dans les locaux, etc. Au final, selon le contexte, ce temps peut être réduit à cinquante, voire à quarante minutes. Mais ce «temps réellement imparti » n'a que peu de sens et doit être rapporté aux besoins des élèves présents dans l'étude. Si chaque élève est autonome face à l'activité prescrite, une durée de quarante minutes est plus que suffisante. Dans ce cas, l'intervenant a le temps de s'engager longuement avec un ou deux élèves dans une démarche centrée sur l'activité. S'il n'est pas inexistant, ce cas reste exceptionnel car nous avons montré plus haut que les difficultés des élèves face à leurs devoirs sont au contraire nombreuses et parfois profondes. Si l'on considère en outre que les élèves les plus fragiles scolairement sont incités à fréquenter régulièrement l'étude, et qu'ils s'y confrontent au moins aux mêmes prescriptions que celles de leurs camarades, on comprend que les besoins des élèves présents dans l'étude sont vastes.

Par ailleurs, l'étude est un moment où le travail des élèves est presque entièrement individualisé. Ce parti pris renvoie à l'idée de «travail personnel » qui caractérise les devoirs (Caillet \& Sembel, 2009), mais aussi plus généralement à une tendance contemporaine visant à chercher dans l'individualisation une réponse aux "besoins spécifiques » des élèves (Frandji $\&$ Rochex, 2011). Cela explique que les groupes d'études soient composés de manière aléatoire, et qu'on puisse y retrouver des élèves de niveaux et de classes différents, et donc un nombre conséquent de prescriptions différentes, qui laisse peu de marges aux intervenants pour réintroduire une forme collective de travail. II résulte de ce parti pris que les intervenants, en présence de douze à quinze enfants au mieux, disposent d'un temps disponible pour chacun qui varie de trois à cinq minutes en moyenne. Ils peuvent difficilement espérer travailler une notion avec un élève en un laps de temps si court. L'ampleur de la tâche est alors en décalage avec la capacité matérielle des intervenants à s'y atteler, et la multitude des sollicitations 
dont ils sont l'objet maintient sur eux une pression qui ne se relâche qu'avec la sonnerie finale.

Nos observations permettent de dégager un castype de l'étude, où les intervenants, lorsqu'ils tentent de mettre en œuvre des situations d'apprentissage, sont accaparés par un élève pendant un temps relativement long par rapport à ce qu'ils peuvent lui consacrer étant donnée la demande pressante de ses camarades. On observe que la queue devant leur table s'allonge, au point parfois que seuls deux ou trois enfants restent assis, ou que les doigts levés se multiplient. L'intervenant qui persiste dans cette démarche voit le groupe se déliter progressivement, et un relatif chahut remplacer l'ambiance de travail indispensable à la réalisation des devoirs. Avec l'agitation croissante émerge pour l'intervenant le risque de perdre le contrôle du groupe. Cette tension permanente et la sensation de ne pas pouvoir réaliser convenablement leur travail revêtent pour les adultes soumis à une telle contrainte un caractère frustrant qu'ils expriment volontiers. Souvent, les enfants ressentent aussi le manque de disponibilité des intervenants et se plaignent de la queue qu'ils doivent faire pour attendre une indication chèrement acquise. II en résulte cette situation paradoxale où l'intervenant manque cruellement de temps, alors que les élèves - pris dans une contradiction entre l'injonction à travailler qui les consigne à leur place dans une posture de travail et leur faible capacité à le faire qui les conduit à demander une aide peu disponible - passent une partie de l'étude à attendre. Les cinquante minutes que dure généralement l'étude peuvent dès lors leur sembler bien longues. Et le mélange d'ennui et de désarroi face à leur travail est rapidement facteur de chahut, ce qui renforce la pression qui pèse sur l'intervenant et diminue en retour sa capacité d'action. L'étude est alors prise dans un cercle vicieux dont il est bien difficile de sortir.

L'adoption par les intervenants d'une démarche centrée sur l'activité justifie l'existence de l'étude parce qu'elle permet de rétablir en partie le flux de la circulation des savoirs entre classe et hors classe. Mais l'engagement dans une telle démarche est très difficile et coûteux. L'énergie que les intervenants doivent déployer pour rassembler les dispositions nécessaires à la mise en place de « situations d'enseignement » est importante, et leur résultat paraît bien peu assuré au vu de leur coût. Nous avons pu les observer lorsque des conditions particulièrement favorables étaient réunies : un intervenant engagé et suffisamment en forme, un élève qu'il s'agit d'aider dont les difficultés sont limitées et la concentration mobilisable, un ensemble de prescriptions dans l'étude comportant peu de difficultés, et des élèves dont l'autonomie face aux apprentissages en jeu permet donc de dégager du temps. Cette situation est rare, et, dans le cas général, les intervenants peuvent être conduits à mettre en œuvre des pratiques moins favorables aux apprentissages pour tenter de réduire les tensions qui pèsent sur leur activité.

\section{Des pratiques d'évitement des situations d'enseignement}

Le recours à ces pratiques, dont certaines semblent contribuer à renforcer l'inscription des élèves dans les logiques de travail les plus improductives sur le plan cognitif, peut surprendre de la part de professionnels. Elles résultent en fait d'un " compromis entre des rationalités multiples " (Goigoux, 2007) et peuvent toutes s'interpréter comme des manières d'éviter les situations d'enseignement, de substituer à une " autonomie face aux apprentissages " que la classe n'a pas permis de construire une " autonomie face aux tâches " nécessaire au fonctionnement de l'étude. Ces pratiques, qui ne sont pas sans évoquer les «stratégies de survie " mises en œuvre par les enseignants dans les classes (Woods, 1997), sont autant de compromis opérés par les adultes pour trouver un « modus vivendi » acceptable.

Le premier type de pratiques d'évitement observé consiste à retarder le dévoilement des besoins de remédiation des élèves, en renvoyant le plus possible ces derniers à leurs propres ressources pour faire leurs devoirs. Ce sont celles qui s'observent le plus à l'étude, sans doute parce qu'elles permettent de ne pas renoncer complètement aux logiques d'enseignement et donc de "sauver les apparences " (Woods, 1997). La mise en place d'un système de validation de l'apprentissage des leçons, qui exige des élèves qu'ils sachent d'abord réciter par cœur leur leçon avant de pouvoir accéder aux questions permettant de vérifier leur degré de compréhension des notions en jeu, relève typiquement de ce premier genre:

Trente minutes après le début de l'étude, Linda (CM1) juge qu'elle connaît sa leçon. Elle s'insère dans la queue qui s'est formée près du bureau de l'intervenant et qui, ce soir-là, est aussi son maître. Son tour arrive. Elle se met à réciter sa leçon. Elle en connaît bien le début mais marque peu après un temps d'hésitation et se trompe sur un mot. L'enseignant l'interrompt aussitôt : "On a jamais parlé de ça cet aprèsmidi ! " II ferme aussitôt son cahier et - tout en invitant du regard le candidat suivant à se présenter - il la congédie sur un ton théâtral : "Va réviser ! " Leur échange a duré moins de deux minutes. 
Ce mode de validation permet de réduire considérablement la masse des besoins susceptibles de s'exprimer : seuls les élèves qui ont franchi préalablement les épreuves de " bas niveau » ont accès à celles de " haut niveau » (Bautier \& Goigoux, 2004). II garantit que les élèves puissent travailler de manière apparemment autonome durant la plus grande partie de l'heure. Mais on ne peut le qualifier de choix pédagogique car il entre en totale contradiction avec les recommandations que formulent les intervenants : « Je vous rappelle que pour apprendre efficacement une leçon, il faut déjà la comprendre. Si vous apprenez les phrases juste bêtement par cœur, ça sert à rien et ce sera plus compliqué pour vous de l'apprendre ", déclare par exemple l'enseignant de Linda ce soir-là. Dans ce système, les élèves les plus en difficulté face à leurs devoirs, qui ont le plus de mal à apprendre par cœur, voient leur temps d'aide se réduire.

Un second type de pratiques d'évitement consiste à valider directement les malentendus patents dont les élèves sont porteurs, sans chercher à les orienter vers des logiques d'apprentissage appropriées. On retrouve ici la tendance à " dégrader " les tâches décrite plus haut :

Deux élèves de CE1 doivent apprendre leur leçon de géométrie sur les polygones. Elles n'en ont manifestement pas compris les enjeux, ni même le sens. Pourtant, l'intervenant se contente par un guidage de plus en plus précis d'en appeler à leur mémorisation du texte. II les conforte dans l'idée qu'apprendre une leçon de géométrie ne consiste qu'à en retenir les termes par cœur et dans l'ordre. II ne vérifie pas si les notions sont comprises. Certaines de ses relances montrent qu'il cherche au contraire à éluder les problèmes notionnels, en particulier la confusion entre la définition de l'objet géométrique et l'acte de dessiner une figure. Le moment de récitation se transforme progressivement en simulacre et se clôt par un : «Voilà, c'est très bien. »

Dans sa forme la plus extrême, ce renoncement aux logiques d'enseignement peut conduire les intervenants à faire les devoirs à la place des enfants, en inscrivant par exemple les bonnes réponses au crayon à papier dans la marge de leurs cahiers tout en leur demandant de les recopier au propre à la maison. Ces pratiques relevant du second type s'observent le plus souvent en fin de séance (notamment quand elles ont été manifestement éprouvantes) et sont beaucoup moins fréquentes que celles du premier genre. Elles semblent correspondre pour les intervenants au compromis le moins satisfaisant.

Le recours à de telles pratiques permet aux adultes de maintenir l'étude « en vie », c'est-à-dire « en ordre » et « en activité » (Goigoux, 2007). Nous avons montré plus haut que le temps d'intervention disponible par élève n'excède pas trois à cinq minutes par séance et que toute forme d'enlisement auprès d'un élève ou d'un groupe d'élèves se traduit systématiquement, à plus ou moins court terme, par une perte de la maîtrise du groupe. Réintroduire des formes factices d'autonomie et réduire par conséquent le temps d'intervention par élève à quelques minutes, fragmentées en plusieurs interactions courtes, permet de conserver une bonne maîtrise du groupe. Cela évite en outre l'émergence d'éventuels conflits de justice liés à l'inégale répartition de l'aide entre élèves, car chacun accède à la part de temps qui lui revient de droit. Les pratiques d'évitement permettent aussi de préserver l'image que les élèves ont d'eux-mêmes, de différer l'expérience potentiellement dévalorisante de l'échec (Boullier, 1984). Elles sont en somme pour les intervenants, qui arrivent à l'étude au terme d'une pleine journée de travail parfois éprouvante, une manière de se préserver. II s'agit de s'épargner la fatigue physique, intellectuelle et morale d'avoir à reprendre (avec peu de chance de parvenir à un résultat satisfaisant) des notions complexes avec les élèves, tout en ayant à faire face à un groupe rapidement amené à se déconcentrer, voire à contester. Le dispositif d'aide aux devoirs est donc susceptible de produire des " effets pervers " dans la mesure où les enseignants sont régulièrement conduits à mettre en œuvre, plus ou moins consciemment, des stratégies d'évitement dont les élèves qui ont le plus besoin d'aide pâtissent le plus.

Si l'externalisation du travail personnel des élèves contribue à la production et à l'accentuation des inégalités sociales de réussite scolaire (Glasman \& Besson, 2004 ; Rayou, 2009 ; Kakpo \& Rayou, 2010 ; Kakpo, 2012), la ré-internalisation des devoirs, même lorsqu'elle permet de les placer sous la responsabilité des enseignants, n'est pas en soi un gage de réduction de l'échec scolaire. L'enquête déconstruit l'idée selon laquelle on pourrait résorber les inégalités d'apprentissage en se bornant - sans jamais interroger le fonctionnement du système - à compenser les « handicaps " dont pâtiraient les enfants des classes populaires et les inégales ressources dont disposent les familles en matière d'encadrement des devoirs. Les effets positifs mais aussi négatifs que peuvent produire les dispositifs d'aide aux devoirs dépendent intrinsèquement de ce qui circule entre classe et dispositifs hors la classe.

L'analyse de notre corpus nous conduit à distinguer trois grands types de " boucles " susceptibles de produire des effets diamétralement opposés. La première est une "boucle vertueuse ", où circulent 
des tâches renvoyant à des enjeux que les élèves se sont suffisamment appropriés en amont pour travailler seuls à l'étude et cela quels que soient leur âge et le degré de leurs performances scolaires. L'exportation de ces tâches ne rend pas pour autant superflue la présence d'intervenants aussi qualifiés que les enseignants à l'étude. Il leur revient en effet de vérifier la bonne acquisition des notions et de consolider ces acquis, autant d'activités pour lesquelles ils peuvent remobiliser utilement leurs habitudes professionnelles. À cette "boucle vertueuse " s'oppose un second type de boucle : la "fausse boucle ", où transitent des prescriptions qui ne semblent pas avoir d'autres buts qu'elles-mêmes et mettent en jeu des tâches de « bas niveau cognitif ". La circulation de ces tâches, qui rend la présence d'intervenants aussi qualifiés que des enseignants assez superflue, est propice à la création d'" effets de leurre " chez les élèves (Bautier \& Goigoux, 2004). Ces tâches se coulent généralement bien dans le moule de l'étude puisqu'elles permettent aux élèves de travailler seuls et aux enseignants d'intervenir auprès d'eux à moindre frais. Le troisième type de boucle est une "boucle contre-productive ", où circulent des tâches qui renvoient bel et bien à des enjeux d'apprentissage mais où ceux-ci n'ont pas été correctement perçus par les élèves. La circulation de ce type de tâches tend à déplacer le centre de gravité du système en transférant vers l'étude les difficultés non résolues en classe. Les élèves s'y révèlent alors peu autonomes face aux apprentissages et la situation exige des intervenants qu'ils s'engagent dans de coûteuses démarches de restauration des notions. Mais ces démarches sont peu adaptées au format du dispositif, qui produit en retour des effets pervers : pour rendre possible le fonctionnement de l'étude, les intervenants sont conduits à mettre en œuvre des pratiques d'« évitement » des situations d'enseignement, qui favorisent peu l'apprentissage voire contribuent à renforcer l'inscription des élèves dans des logiques contraires aux exigences scolaires à long terme. Convertir cette boucle en une «boucle vertueuse " relève donc pour les intervenants de l'exploit et exige la conjonction de conditions rarement réunies. Dès lors, il n'est pas rare, en fin de séance, de les voir inciter les élèves à retravailler le soir à la maison et à solliciter pour cela l'aide d'un membre de leur famille.

Au terme de cette enquête exclusivement qualitative, nous ne sommes bien évidemment pas en mesure de pouvoir quantifier rigoureusement ce que représente la part de chacune de ces boucles. Nous pouvons simplement affirmer que la "boucle contre-productive " nous semble être à l'œuvre de manière récurrente. Plutôt qu'un dispositif de lutte contre l'échec scolaire, l'étude nous apparaît surtout à ce jour comme une caisse de résonance voire d'amplification des difficultés non résolues au sein de la classe. On peut en revanche se demander si les dispositifs de ce genre ne constituent pas pour l'institution scolaire un moyen contemporain privilégié de se dédouaner de la responsabilité de l'échec scolaire et de la faire endosser aux élèves et à leur famille. À l'heure où un consensus politique semble se dégager en faveur de la ré-internalisation du travail personnel des élèves à tous les niveaux du système éducatif, il semble important de rappeler qu'un dispositif d'aide aux devoirs n'est pas une composante autonome du monde scolaire et qu'une "réforme des devoirs " ne peut avoir de sens qu'articulée à une réflexion de fond portant aussi bien sur les pratiques pédagogiques de la classe que sur le fonctionnement de l'ensemble du système éducatif.

Séverine Kakpo severinekakpo@gmail.com Université Paris 8-Vincennes-Saint-Denis, CIRCEFT-ESCOL

Julien Netter juliennetter@free.fr Université Paris 8-Vincennes-Saint-Denis, CIRCEFT-ESCOL 


\section{NOTES}

1 Cette recherche a été commanditée par la commune de Granville. Elle a donné lieu à la publication d'un rapport : S. Bonnéry \& S. Kakpo (dir.) (2012). Circulation des savoirs et des dispositifs entre classe et dispositifs hors la classe. CIRCEFT-ESCOL, Paris 8-VincennesSaint-Denis. La première partie du rapport, qui traite des dispositifs d'aide aux devoirs, a été rédigée par nous-mêmes. Tout au long de cette recherche, nous avons bénéficié des éclairages d'É. Bautier, S. Bonnéry, B. Marin et P. Rayou ainsi que de la contribution de V. Ardoin, M. Marnet et M. van Brederode. Qu'ils en soient remerciés ici.

2 Dans ce texte, le mot " étude " renvoie à « dispositif d'aide aux devoirs ».

3 II accueille dans les locaux de l'école des groupes d'élèves issus de différentes classes. Les enfants y sont inscrits à la demande des parents, parfois incités par les enseignants. Ils fréquentent l'étude deux soirs par semaine en moyenne, quatre soirs de façon plus exceptionnelle. Les séances durent environ cinquante minutes.

4 Nous nous référons ici à des documents de cadrage internes. La suite de l'article montre pourquoi les écoles n'adoptent pas dans les faits le mode d'organisation préconisé.
5 C'est ce que se propose de faire J. Netter dans le cadre de la thèse qu'il conduit depuis 2011 sous la direction de P. Rayou : La division du travail scolaire. Devoirs et inégalités d'apprentissage.

6 Celles-ci étaient au nombre de quatre.

7 Expression qui figure dans les documents de cadrage.

8 II n'est pas toujours facile de quantifier le nombre de tâches, et nous avons parfois été conduits à considérer qu'un exercice particulièrement long pouvait être considéré comme deux tâches. De même, pour les leçons, nous avons tenté de constituer une sorte de " tâche étalon » en fonction des quantités généralement prescrites (par exemple pour les nombres de mots dont il faut mémoriser l'orthographe ou le nombre de tables de multiplications à apprendre).

9 L'enquête montre que les enseignants ne se conforment généralement pas aux préconisations officielles, qui interdisent les devoirs écrits en primaire.

10 Cf. supra pour le détail sur les modalités de recueil.

11 Nous nous référons ici aux documents de cadrage du dispositif et donc aux modes institutionnels de catégorisation des élèves.

\section{BIBLIOGRAPHIE}

ARMAND A. \& GILLE B. (2006). La contribution de l'éducation prioritaire à l'égalité des chances des élèves. Rapport au ministre de l'Éducation nationale, de l'Enseignement supérieur et de la Recherche, n० 2006-075.

BARRÈRE A. (2003). Travailler à l'école. Que font les élèves et les enseignants du secondaire? Rennes: Presses universitaires de Rennes.

BAUTIER É., CRINON J., DELARUE-BRETON C. \& MARIN B. (2012). "Les textes composites: des exigences de travail peu enseignées ? ". Repères, n॰ 45, p. 63-79.

BAUTIER É. \& GOIGOUX R. (2004). « Difficultés d'apprentissage, processus de secondarisation et pratiques enseignantes: une hypothèse rationnelle ". Revue française de pédagogie, no 148, p. 89-100.

BAUTIER É. (2010). «Changements curriculaires : des exigences contradictoires qui construisent des inégalités. Entre littéracie étendue, segmentation et contextualisation des savoirs ". In Choukri Ben Ayed (dir.), L'école démocratique. Vers un renoncement politique ? Paris : Armand Colin, p. 83-93.

BAUTIER É. \& RAYOU P. (2009). Les inégalités d'apprentissage. Programmes, pratiques et malentendus scolaires. Paris : PUF.

BAUTIER É. \& ROCHEX J.-Y. (2007). « Ces malentendus qui font les différences ». In J. Deauvieau \& J.-P. Terrail (dir.), Les sociologues, l'école et la transmission des savoirs. Paris : La Dispute, p. 227-241.

BAUTIER É., BONNERY S. \& KAKPO S. (2013). « D'hier à aujourd'hui, les manuels à l'école élémentaire et au collège ». In S. Bonnéry (dir.), Supports pédagogiques et inégalités scolaires. Études sociologiques. Paris : La Dispute (sous presse).

BERNSTEIN B. (1975). Langage et classes sociales. Codes socio-linguistiques et contrôle social. Paris : Éd. de Minuit.
BERNSTEIN B. (2007). Pédagogie, contrôle symbolique et identité. Théorie, recherche, critique. Laval : Presses de l'université Laval.

BONNÉRY S. (2007). Comprendre l'échec scolaire. Élèves en difficultés et dispositifs pédagogiques. Paris : La Dispute.

BONNÉRY S. (2009). "Scénarisation des dispositifs pédagogiques et inégalités d'apprentissage ". Revue française de pédagogie, no 167, p. 13-24.

BOULLIER D. (1984). "Une expérience d'entraide scolaire à Rennes : différer l'échec scolaire ». Sauvegarde de l'enfance, no 4, p. 475-498.

BROSSARD M. (1993). "Un cadre théorique pour aborder l'étude des élèves en situation scolaire ». Enfance, n० 46(2), p. 189-200.

BROSSARD M. (1994). L'adaptation de l'enfant à l'école. Paris : Stablon.

CAILLET V. \& SEMBEL N. (2009). "Points de vue et pratiques des partenaires du travail hors la classe ». In P. Rayou (dir.), Faire ses devoirs, enjeux cognitifs et sociaux d'une pratique ordinaire. Rennes: Presses universitaires de Rennes.

CHARTIER A.-M. (2003). «Exercices écrits et cahiers d'élèves : réflexions sur des pratiques de longue durée ». Le Télémaque, no 24, p. 81-110.

CHARTIER A.-M. \& RENARD P. (2000). "Cahiers et classeurs : les supports ordinaires du travail scolaire ». Repères, no 22, p. 135-160.

CHAUVEAU G. (1999). « Les ZEP, entre discrimination et discrimination positive ». Mouvements, no 5, p. 62-70.

CLOT Y. \& FAÏTA D. (2000). « Genre et style en analyse du travail ». Travailler, n० 4, p. 7-42.

FÉLIX C. (2002). Une étude comparative des gestes de l'étude personnelle : le cas des mathématiques et de l'histoire. Thèse, sciences de l'éducation, université Aix-Marseille 1. 
FÉLIX C., SAUJAT F. \& COMBES C. (2012). « Des élèves en difficulté aux dispositifs d'aide : une nouvelle organisation du travail enseignant? ». Recherches en éducation, hors série no 4, p. 19-30.

FRANDJI D. \& ROCHEX J.-Y. (2011). « De la lutte contre les inégalités à l'adaptation aux "besoins spécifiques" ". Recherches et formations, no 80, p. 95-108.

GLASMAN D. (2001). L'accompagnement scolaire. Sociologie d'une marge de l'école. Paris: PUF.

GLASMAN D. \& BESSON L. (2004). Le travail des élèves pour l'école en dehors de l'école. Rapport pour le Haut conseil de l'évaluation de l'école.

GOIGOUX R. (2007). "Un modèle d'analyse de l'activité des enseignants ». Éducation \& didactique, vol. 1, n॰ 3 , p. 47-70.

KAKPO S. (2012). Les devoirs à la maison. Mobilisation et désorientation des familles populaires. Paris : PUF.

KAKPO S. \& RAYOU P. (2010). "Contrats didactiques et contrats sociaux du travail hors la classe ». Éducation \& didactique, vol. 4 , n०2, p. 7-24.

LAHIRE B. (2001). "La construction de l'“autonomie" à l'école primaire : entre savoirs et pouvoirs ". Revue française de pédagogie, no 135, p. 151-161.

LAHIRE B. (1993). Culture écrite et inégalités scolaires. Sociologie de l'« échec scolaire "à l'école primaire. Lyon : Presses universitaires de Lyon.

PERRENOUD P. (1995). Métier d'élève et sens du travail scolaire. Paris : ESF.

MOISAN C. \& SIMON J. (1997). Les déterminants de la réussite scolaire en zone d'éducation prioritaire. Paris : INRP.

PIQUÉE C. (2001). Gérer les inégalités de réussite à l'école primaire : public, modes de fonctionnement et efficacité pédagogique des dispositifs d'accompagnement scolaire. Thèse de doctorat, sciences de l'éducation, université de Dijon.

PIQUÉE C. (2003). «Public, modes de fonctionnement et efficacité pédagogique de l'accompagnement à la scolarité ". Ville-École-Intégration, no 132, p. 188-203
RAYOU P. (dir.) (2009). Faire ses devoirs, enjeux cognitifs et sociaux d'une pratique ordinaire. Rennes: Presses universitaires de Rennes.

REUTER Y. (2007). "La conscience disciplinaire ». Éducation \& didactique, vol. 1, n ${ }^{\circ} 2$, p. 57-71.

ROCHEX J.-Y. (1997). « Les ZEP : un bilan décevant ». In J.-P. Terrail (dir.), La scolarisation de la France : critique de l'état des lieux. Paris : La Dispute, p. 123-139.

ROCHEX J.-Y. (2010). "Les trois "âges" des politiques d'éducation prioritaire : une convergence européenne ? ". In C. Ben Ayed (dir.), L'école démocratique. Vers un renoncement politique ? Paris : Armand Colin, p. 94-108.

ROCHEX J.-Y. \& CRINON J. (dir.) (2011). La construction des inégalités scolaires. Au cœur des pratiques et des dispositifs d'enseignement. Rennes : Presses universitaires de Rennes.

SUCHAUT B. (2005). " Regard sur l'efficacité des dispositifs de lutte contre l'échec scolaire ». Regards sur l'actualité, n³10, p. 51-58.

THIN, D. (1998). Quartiers populaires. L'école et les familles. Lyon: Presses universitaires de Lyon.

VIGNER G. (1997). "La représentation du savoir : mise en page et mise en texte dans les manuels scolaires". Cahiers du français contemporain, no 4, p 47-82.

VYGOTSKY L. ([1934] 1985). Pensée et langage. Paris : Éd. Sociales.

WOODS P. (1997). " Les stratégies de survie des enseignants ». In J.-C. Forquin (dir.), Les sociologues de l'éducation américains et britanniques. Bruxelles: De Boeck ; Paris : INRP, p. 351-376.

ZAKARIA H. (2012). Que font les maitres ? Pour un bilan de la rénovation pédagogique à l'école. Paris: La Dispute.

ZAKHARTCHOUK J.-M. (2000). "Les consignes au cœur de la classe : geste pédagogique et geste didactique ». Repères, $\mathrm{n}^{\circ} 22$, p. 61-81.

ZERBATO-POUDOU M.-T. (2001). « Spécificités de la consigne à l'école maternelle et définition de la tâche ». Pratiques, no 111-112, p. 115-129. 